\title{
A Computational Investigation of the Finite-Time Blow-Up of the 3D Incompressible Euler Equations Based on the Voigt Regularization
}

\author{
Adam Larios, ${ }^{1}$ Mark R. Petersen, ${ }^{2}$ Edriss S. Titi, ${ }^{3}$ and Beth Wingate ${ }^{4}$ \\ ${ }^{1}$ University of Nebraska-Lincoln* \\ ${ }^{2}$ Los Alamos National Lab ${ }^{\dagger}$ \\ ${ }^{3}$ Texas A\&M University and Weizmann Institute of Science ${ }^{\ddagger}$ \\ ${ }^{4}$ University of Exeter ${ }^{\S}$ \\ (Dated: December 23, 2015)
}

\begin{abstract}
We report the results of a computational investigation of two recently proved blow-up criteria for the 3D incompressible Euler equations. These criteria are based on an inviscid regularization of the Euler equations known as the 3D Euler-Voigt equations. The latter are known to be globally well-posed. Moreover, simulations of the 3D Euler-Voigt equations also require less resolution than simulations of the 3D Euler equations for fixed values of the regularization parameter $\alpha>0$. Therefore, the new blow-up criteria allow one to gain information about possible singularity formation in the 3D Euler equations indirectly; namely, by simulating the better-behaved 3D Euler-Voigt equations. The new criteria are only known to be sufficient criteria for blow-up. Therefore, to test the robustness of the inviscid-regularization approach, we also investigate analogous criteria for blow-up of the 1D Burgers equation, where blow-up is well-known to occur.
\end{abstract}

Keywords: Euler-Voigt, Navier-Stokes-Voigt, Inviscid Regularization,Turbulence Models, $\alpha$-Models, BlowUp Criterion for Euler.

\section{INTRODUCTION}

The 3D Euler equations for incompressible inviscid fluid flow are a source of much mathematical and scientific interest. In particular, these equations exhibit many of the same difficulties as the 3D Navier-Stokes equations in the case of large Reynolds numbers. The question of whether these equations develop a finite-time singularity remains an extremely challenging open problem.

Two new blow-up criteria for the 3D Euler equations for ideal incompressible flow were reported in $[1,2]$. These criteria are of a different character than, e.g., the well-known Beale-Kato-Majda criterion [3]. Traditional computational searches for blow-up seek to identify singularities by analyzing the vorticity coming from the 3D Euler equations themselves, which are not known to be globally well-posed, and moreover, are extremely difficult to simulate accurately. In contrast, the blow-up criteria in $[1,2]$ only rely on analyzing the vorticity of the 3D Euler-Voigt equations, which are globally well-posed and are less computationally intensive to simulate.

An important aspect of the Euler-Voigt model, when used as a regularization for the Euler equations, is that the regularization is inviscid in the sense that it does not add artificial viscosity. Hence, we refer to the Voigtregularization as an inviscid regularization. Moreover, the Voigt-regularization can be used to stabilize simulations of the Euler equations by a method different from adding artificial viscosity, as is done, e.g., in LES (LargeEddy Simulation) models (see, e.g., [4], and the references therein). Inviscid regularization is distinct from regularizations that use artificial viscosity: while artificial viscosity removes energy from the system, the EulerVoigt equations conserve a modified energy for all time (see (I.2) below). We use this conservation as one test of the validity of our simulations. Moreover, the two blowup criteria we test are derived from (I.2) and the shorttime energy conservation of the 3D Euler equations.

In this article, we describe the first computational search for blow-up of the 3D Euler equations based on the criteria in [1] and [2]. The key result of the present work is that, using this approach, we find numerical evidence for the development of a singularity in the 3D Euler equations; however, the purpose of this work is chiefly to motivate the fluid mechanics computational community for further investigation of these criteria, rather than to make a definite claim about blow-up. Because this is a new approach to studying blow-up, we show how the method provides evidence for blow-up in a case where blow-up is well understood; namely, in Burgers equation.

The Euler-Voigt equations were proposed as an inviscid regularization of the Euler equations in [5], where they were first studied. Their viscous counterpart, called the Navier-Stokes-Voigt equations, were studied much earlier in $[6,7]$. The Euler-Voigt equations are given by

$$
\left\{\begin{aligned}
-\alpha^{2} \partial_{t} \nabla^{2} \mathbf{u}+\partial_{t} \mathbf{u}+(\mathbf{u} \cdot \nabla) \mathbf{u}+\nabla p & =0, \\
\nabla \cdot \mathbf{u} & =0, \\
\mathbf{u}(\mathbf{x}, 0) & =\mathbf{u}_{0}(\mathbf{x}) .
\end{aligned}\right.
$$

Here $\alpha>0$ is a regularization parameter having units of length. Note that the usual incompressible Euler equations are formally obtained by setting $\alpha=0$. The unknowns are the fluid velocity field $\mathbf{u}(\mathbf{x}, t)=\left(u_{1}, u_{2}, u_{3}\right)$, and the fluid pressure $p(\mathbf{x}, t)$, where $\mathbf{x}=\left(x_{1}, x_{2}, x_{3}\right)$, and $t \geq 0$. In the present work, we consider only the case of periodic boundary conditions, and we assume that the spatial average of $\int_{\Omega} \mathbf{u}_{0}(\mathbf{x}) d \mathbf{x}=0$, which with (I.1a) 
implies $\int_{\Omega} \mathbf{u}(\mathbf{x}, t) d \mathbf{x}=0$ for all $t$. We denote by $\mathbf{u}^{\alpha}$ the solution to (I.1), and by $\mathbf{u}$ a solution to the Euler equations, both starting from the same initial condition $\mathbf{u}_{0}$. In addition, we denote the corresponding vorticities $\boldsymbol{\omega}:=\nabla \times \mathbf{u}$, and also $\boldsymbol{\omega}^{\alpha}:=\nabla \times \mathbf{u}^{\alpha}$.

System (I.1) was introduced in [5], where existence and uniqueness of solutions was proven for all times $t \in(-\infty, \infty)$. The Euler-Voigt and Navier-Stokes-Voigt equations have been studied analytically and extended in a wide variety of contexts (see, e.g., [1, 5-18], and the references therein). The first computational study of the Navier-Stokes-Voigt and MHD-Voigt equations was carried out in [19]. A recent computational study [20] studied the energy spectrum and other properties of the Euler-Voigt equations. Energy decay for Navier-StokesVoigt was studied in [21].

In [5], the following " $\alpha$-energy" equality was rigorously shown to hold for solutions of (I.1)

$$
\left\|\mathbf{u}^{\alpha}(t)\right\|_{L^{2}}^{2}+\alpha^{2}\left\|\nabla \mathbf{u}^{\alpha}(t)\right\|_{L^{2}}^{2}=\left\|\mathbf{u}_{0}\right\|_{L^{2}}^{2}+\alpha^{2}\left\|\nabla \mathbf{u}_{0}\right\|_{L^{2}}^{2} .
$$

One aim of this paper is to investigate the connection between the Euler equations and Euler-Voigt equations as $\alpha \rightarrow 0$. In [1], it was shown that, for sufficiently smooth initial data, on the time interval $[0, T]$ of existence and uniqueness for strong solutions of the Euler equations, the following estimate holds:

$$
\left\|\mathbf{u}^{\alpha}(t)-\mathbf{u}(t)\right\|_{L^{2}} \leq C \alpha\left(e^{C t}-1\right)^{1 / 2}, \text { for } t \in[0, T] .
$$

where the constant $C$ depends on $\|\mathbf{u}\|_{L^{\infty}\left(0, T ; H^{3}\right)}$. In particular, as $\alpha \rightarrow 0$, solutions to (I.1) converge to the solution the Euler equations in the $L^{\infty}\left([0, T] ; L^{2}\right)$ norm. Combining this with (I.2) and the equality $\|\mathbf{u}(t)\|_{L^{2}}=$ $\left\|\mathbf{u}_{0}\right\|_{L^{2}}$, which holds on $[0, T]$, it was proved in [1], by contradiction, that if

$$
\sup _{t \in\left[0, T^{*}\right]} \limsup _{\alpha \rightarrow 0^{+}} \alpha\left\|\nabla \mathbf{u}^{\alpha}(t)\right\|_{L^{2}}>0,
$$

or, (shown later in [2]) if

$$
\limsup _{\alpha \rightarrow 0^{+}}\left(\alpha \sup _{t \in\left[0, T^{*}\right]}\left\|\nabla \mathbf{u}^{\alpha}(t)\right\|_{L^{2}}\right)>0
$$

then the 3D Euler equations must develop a singularity at or before time $T^{*}$. It was observed in [2] that (I.3) implies (I.4), and hence (I.4) is a stronger criterion than (I.3), i.e., singularities indicated by (I.3) will also be indicated by (I.4). Below, we explore the nature of blow-up in relation to both (I.3) and (I.4).

Since it is unknown whether the 3D Euler equations become singular in a finite interval of time, several criteria for the blow-up of solutions have arisen in the literature, e.g., [3, 22-26]. Perhaps the most celebrated is the Beale-Kato-Majda criterion [3] which states that the solution is non-singular on $[0, T]$ if and only if

$$
\int_{0}^{T}\|\boldsymbol{\omega}(t)\|_{L^{\infty}} d t<\infty .
$$

Hence, in many computational searches for blow-up of solutions of the Euler equations (see, e.g., [27-31], and references therein), $\|\boldsymbol{\omega}(t)\|_{L^{\infty}}$ is the main quantity of interest. Furthermore, periodic boundary conditions are often used in computational studies (the review [32] cites more than twenty such studies). Thanks to the identity $\|\nabla \mathbf{v}\|_{L^{2}}=\|\nabla \times \mathbf{v}\|_{L^{2}}$, holding for all smooth divergencefree functions $\mathbf{v}$, one can view (I.3) and (I.4) as conditions on the vorticity $\boldsymbol{\omega}^{\alpha}$ of the Euler-Voigt equations.

Remark I.1. We emphasize that quantity (I.5) is computed from solutions of the 3D Euler equations, which are not known to be globally well-posed. In contrast, the quantity $\left\|\nabla \mathbf{u}^{\alpha}\right\|_{L^{2}}$ in (I.3) and (I.4) is computed from solutions to (I.1), which is known to be well-posed globally in time. This is important because it allows us the possibility of computing measures of blow up in numerical simulations, where we only have finite resolution.

It is thus of interest to investigate the dependence of $\left\|\nabla \mathbf{u}^{\alpha}(t)\right\|_{L^{2}}$ on $\alpha$ and $t$, for some given initial data, as $\alpha \rightarrow 0$. We do this computationally in Section III. It is unknown if (I.3) or (I.4) are necessary conditions for the blow-up of solutions of the 3D Euler equations. Hence, to further support the notion that blow-up may be indicated by (I.3) or (I.4), we consider the 1D inviscid Burgers equation, which has solutions that blow up in finite time. In Section IV, we apply a Voigt-type regularization to the 1D Burgers equation (yielding the BBM equation (IV.1)), and show computationally that the analogues of (I.3) and (I.4) appear to be satisfied when $T^{*}$ approaches the blowup time of the Burgers equation.

\section{NUMERICAL METHODS}

All simulations were carried out using a pseudospectral method on the periodic unit cube; namely, with derivatives computed in Fourier space, and products computed in physical space with the $2 / 3$ 's dealiasing rule applied. Time stepping was done using a fully-explicit fourthorder Runge-Kutta-4 scheme resepecting the advective CFL condition. The pressure was computed explicitly by the standard Chorin-Temam projection method $[33,34]$. For the Euler-Voigt simulations, Taylor-Green initial data was used on the domain $[0,1]^{3}$, namely,

$$
\begin{aligned}
& u_{1}=\sin (2 \pi x) \cos (2 \pi y) \cos (2 \pi z), \\
& u_{2}=-\cos (2 \pi x) \sin (2 \pi y) \cos (2 \pi z), \quad u_{3}=0 .
\end{aligned}
$$

See, e.g., [35, 36]) for classic computational studies on the Euler equations with Taylor-Green initial data.

It is important for this study that the energy and the enstrophy are properly captured. Therefore, we consider the maximum relative error in the $\alpha$-energy by

$$
\max _{t \in[0, T]} \frac{\left\|\mathbf{u}^{\alpha}(t)\right\|_{L^{2}}^{2}+\alpha^{2}\left\|\nabla \mathbf{u}^{\alpha}(t)\right\|_{L^{2}}^{2}-\left\|\mathbf{u}_{0}\right\|_{L^{2}}^{2}-\alpha^{2}\left\|\nabla \mathbf{u}_{0}\right\|_{L^{2}}^{2}}{\left\|\mathbf{u}_{0}\right\|_{L^{2}}^{2}+\alpha^{2}\left\|\nabla \mathbf{u}_{0}\right\|_{L^{2}}^{2}} .
$$


Due to the Runge-Kutta-4 time-stepping, perfect $\alpha$-energy conservation is not expected. However, every Euler-Voigt simulation at resolution $1024^{3}$ reported in this article had a relative $\alpha$-energy error of no more than $2.7 \times 10^{-8}$ over the time interval of integration. In FIG. II.1, one can see the typical behavior of the terms comprising the $\alpha$-energy (I.2), with a monotonic transfer of the energy $\left(\left\|\mathbf{u}^{\alpha}\right\|_{L^{2}}^{2}\right)$ to the enstrophy $\left(\alpha^{2}\left\|\nabla \mathbf{u}^{\alpha}\right\|_{L^{2}}^{2}\right)$.

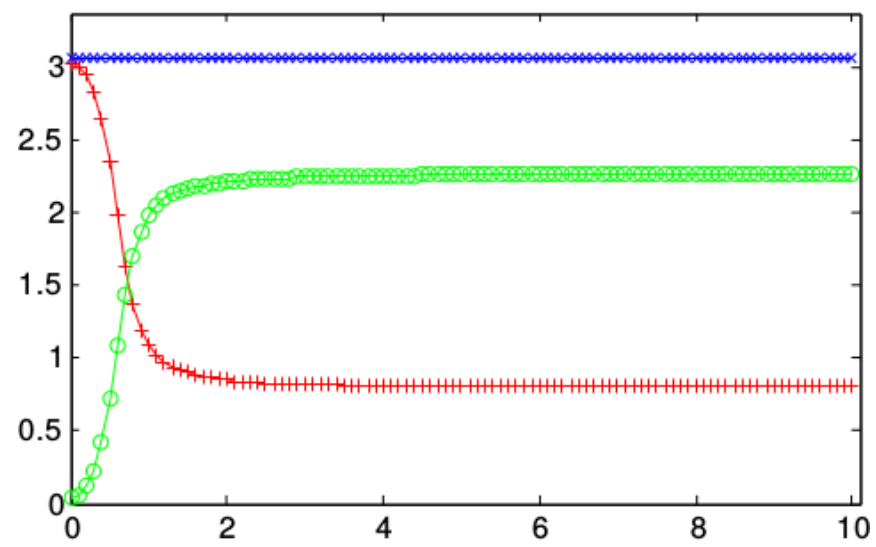

FIG. II.1. Energy and enstrophy (scaled by $\alpha^{2}$ ) vs. time for the 3D Euler-Voigt equations. (blue " $\times$ ": $\left\|\mathbf{u}^{\alpha}(t)\right\|_{L^{2}}^{2}+\alpha^{2}\left\|\nabla \mathbf{u}^{\alpha}(t)\right\|_{L^{2}}^{2}$, green "o": $\left\|\mathbf{u}^{\alpha}(t)\right\|_{L^{2}}^{2}$, red “+": $\alpha^{2}\left\|\nabla \mathbf{u}^{\alpha}(t)\right\|_{L^{2}}^{2}$.) Resolution: $128^{3}$.

Remark II.1. We emphasize that, since (I.1) is globally well-posed in time, we are allowed to integrate the equations beyond the point of possible singularity for the 3D Euler equations. That is, if the Euler equations develop a singularity at time $T^{*}$, for given initial data, we may safely integrate (I.1) with the same initial data up to and beyond $T^{*}$. We believe this to be a major distinction of the blow-up criteria (I.3) and (I.4) from other blow-up criteria for the 3D Euler equations, such as (I.5).

\section{SINGULARITY DETECTION}

In this section, we computationally investigate the blow-up criteria (I.3) and (I.4). We simulate solutions of (I.1) with initial data (II.1), tracking the quantity

$$
\alpha\left\|\nabla \mathbf{u}^{\alpha}(t)\right\|_{L^{2}} \equiv \alpha\left\|\boldsymbol{\omega}^{\alpha}(t)\right\|_{L^{2}},
$$

for several values of $t$, as $\alpha \rightarrow 0$. To investigate (I.3) computationally, we track the growth rate of the quantity in (III.1) as a function of $t$ and $\alpha$, shown in FIG. IV.1 as contours of constant $t$.

If, for any given $t=T^{*}>0$, a non-positive slope persists as $\alpha \rightarrow 0$, then (I.3) must follow, indicating that the 3D Euler equations develop a singularity on the interval $\left[0, T^{*}\right]$. In FIG. IV.1, we see that the contours corresponding to $t \geq 1.6$ indeed have a non-positive slope for small $\alpha$, indicating that $\log \left(\alpha\left\|\nabla \mathbf{u}^{\alpha}(t)\right\|_{L^{2}}\right) \geq c$ for some $c>0$ and $t \geq 1.6$. Therefore, $\alpha\left\|\nabla \mathbf{u}^{\alpha}(t)\right\|_{L^{2}} \geq e^{c}$, indicating that (I.3) may hold. We therefore consider this to be evidence of a possible blow-up in the Euler equations with initial data (II.1) at or before $T^{*}=1.6$. Of course, as $\alpha \rightarrow 0$, the resolution must increase to infinity for the simulation to be well-resolved. Thus, we are always limited by some $\alpha_{\min }>0$, which is determined by computational resources.

We next investigate the blow-up criterion given by (I.4). Let us make an ansatz that

$$
\sup _{t \in\left[0, T^{*}\right]}\left\|\nabla \mathbf{u}^{\alpha}(t)\right\|_{L^{2}} \sim \mathcal{O}\left(\alpha^{p}\right)
$$

for $T^{*}>0$ sufficiently large and for some power $p$, possibly depending on $T^{*}$. If $p \leq-1$, then (I.4) holds. The quantity in (III.2) is shown in FIG. IV.2 as a function of $\alpha$ with various values of $T^{*}$. The slope of the lines corresponding to $T^{*} \geq 1.6$ remain strictly less than -1 for small $\alpha$, indicting again a possible blow-up of the Euler equations somewhere on the time interval $[0,1.6]$.

\section{BLOW-UP FOR BURGERS VIA THE BENJAMIN-BONA-MAHONY EQUATIONS}

In this section, we consider the 1D Benjamin-BonaMahony (BBM) equation for water waves, given by

$$
-\alpha^{2} u_{t x x}+u_{t}+u u_{x}=0, \quad u(x, 0)=u_{0}(x) .
$$

This equation was derived in [37] as a model for water waves, where it was shown to be globally well-posed. It can be viewed as a regularization of the inviscid Burgers equation by formally setting $\alpha=0$ in (IV.1). Notably, we do not propose here that the solution of (IV.1) converges to the unique entropy solution of Burgers equation. We view this equation as a 1D analogue of the Euler-Voigt equations, with a crucial difference being that the pressure and the divergence-free condition are absent. One advantage of considering equation (IV.1) is that that solutions to the Burgers equation are known to develop a singularity in finite time; a fact that is unknown for solutions of the 3D Euler equations. By following arguments similar to those in [1], it is straight-forward to show that the analogues of both (I.3) and (I.4) imply blow-up for the Burgers equation on $\left[0, T^{*}\right]$.

We use the method described in Section III to try to identify the known singularity in Burgers equation $\left(u_{t}+\right.$ $\left.u u_{x}=0\right)$. That is, we test the analogues of criteria (I.3) and (I.4) for problem (IV.1), as $\alpha \rightarrow 0$. The domain is the periodic interval $[-\pi, \pi]$, and the initial data is $u_{0}(x)=-\sin (x)$. The solution of Burgers equation with this initial data develops a singularity at time $T^{*}=1$.

FIG. IV.3 and FIG. IV.4 are analogous to FIG. IV.1 and FIG. IV.2, respectively. In FIG. IV.3, before the (Burgers) blow-up time $T^{*}=1$, the curves tend to zero, 


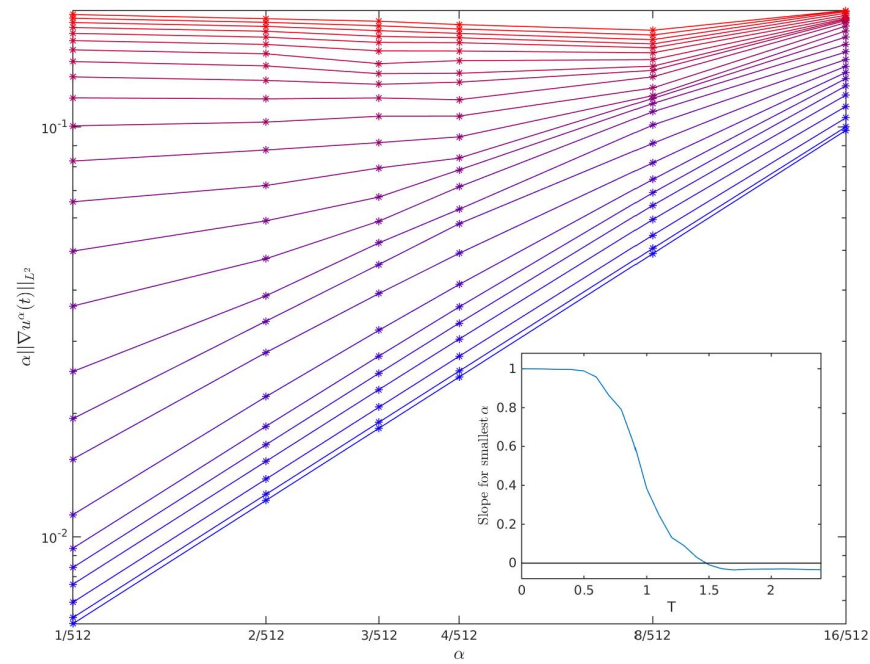

FIG. IV.1. Log-log plot of $\alpha$ vs. $\alpha\left\|\nabla \mathbf{u}^{\alpha}(t)\right\|_{L^{2}}$ for 3D EulerVoigt equations at $t=0.0,0.1,0.2,0.3, \ldots, 2.3,2.4$. Initial condition is blue; plots drawn increasingly red as time increases. Inset: slope, with cross-over time at $T \approx 1.5$. Resolution: $1024^{3}$.

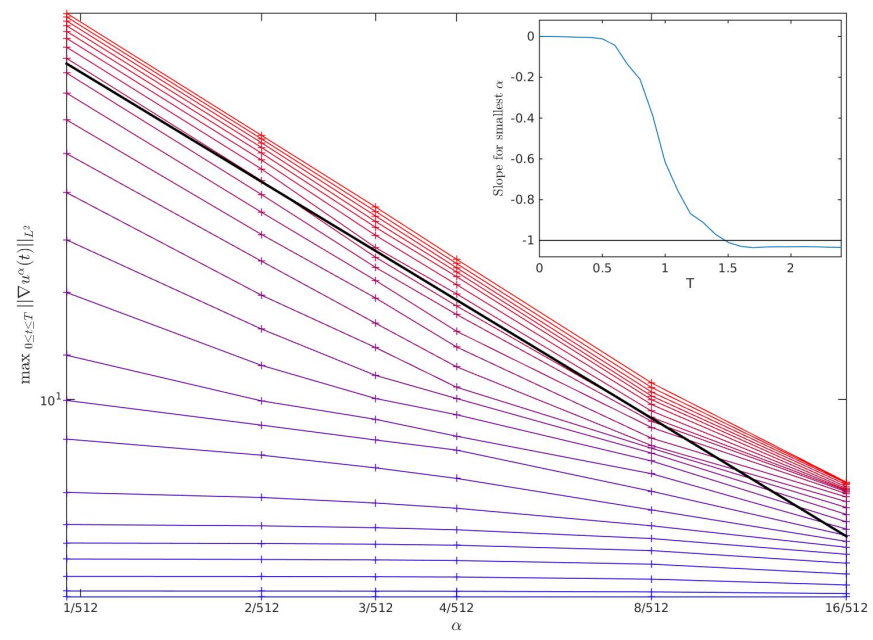

FIG. IV.2. Log-log plot of $\max _{t \in\left[0, T^{*}\right]}\left\|\nabla \mathbf{u}^{\alpha}(t)\right\|_{L^{2}}$ for the 3D Euler-Voigt equations with same times and coloring as FIG. IV.1. Inset: slope, with cross-over time at $T \approx \approx 1.5$. Thick line is $\alpha$ vs. $C \alpha^{-1}$. Resolution: $N^{3}=1024^{3}$.

obeying a uniform power-law. However, slightly after $T=1.0$, the curves become convex on the log-log plot for small $\alpha$. If this trend continues as $\alpha \rightarrow 0$, the analogue of criterion (I.3) implies Burgers equation develops a singularity at or before time $T^{*}=1$. This is already known by other means (e.g., the method of characteristics), but the result here serve to corroborate criterion (I.3) as a test for blow-up. Similarly, FIG. IV.4 indicates a blow-up near $T=1$, via criterion (I.4).

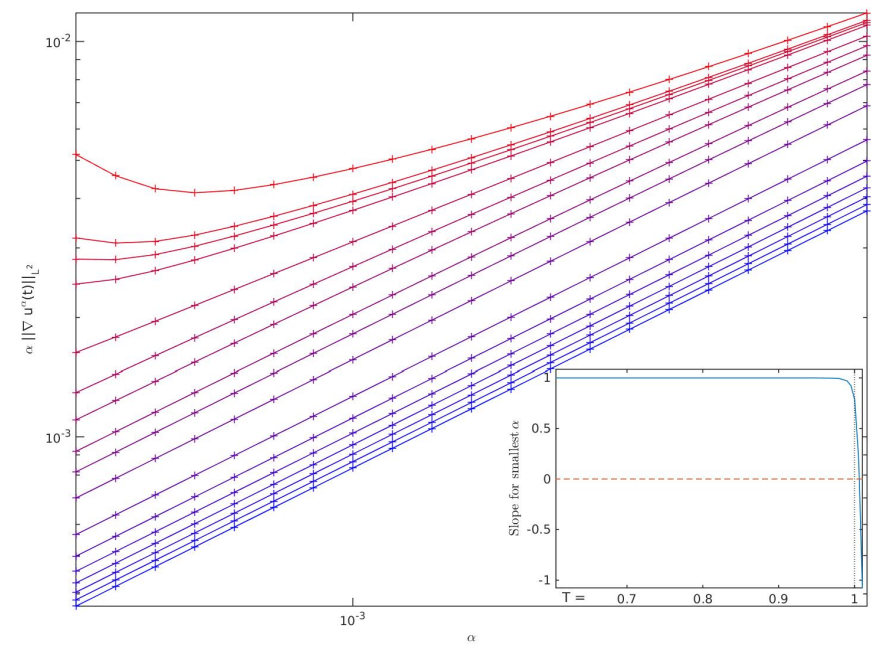

FIG. IV.3. Log-log plot of $\alpha$ vs. $\alpha\left\|u_{x}^{\alpha}(t)\right\|_{L^{2}}$ for the BBM equations at $t=0.6,0.65,0.7,0.75,0.8,0.85,0.9,0.95,0.97$, $0.98,0.99,0.995,1,1.005,1.006,1.007,1.01$. Inset: Slope near smallest $\alpha$-values becomes negative at $T \approx 1.006$, indicating a blow-up at or before this time. Resolution: $N=8192$.

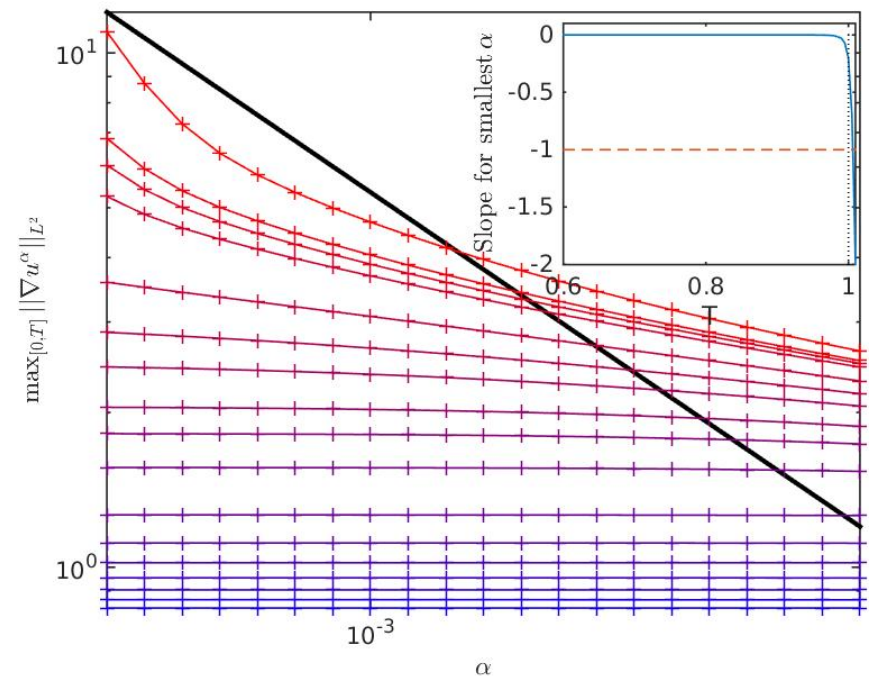

FIG. IV.4. Log-log plot of $\alpha$ vs. $\max _{0 \leq t \leq T}\left\|\nabla \mathbf{u}^{\alpha}(t)\right\|$ for the BBM equations at various values of $\mathrm{T}$. Same $T$ values as $t$ values in FIG. IV.3. Thick line is $\alpha$ vs. $C \alpha^{-1}$. Inset: Slope near smallest $\alpha$-values drops below -1 at $T \approx 1.006$, indicating a blow-up at or before this time. Resolution: $N=8192$.

\section{CONCLUSION}

The results in Section III provide computational evidence for the development of a singularity of the 3D Euler equations with Taylor-Green initial data (II.1), at or before time $T=1.6$. Future studies at smaller $\alpha$-values (and thus higher resolution) may either corroborate or contradict these findings. In any case, the approach presented here represents a new method in the computational search for singularities, and its effectiveness has been demonstrated in the case of Burgers equation. 


\section{ACKNOWLEDGMENTS}

The work of E.S.T was supported in part by ONR grant number N00014-15-1-2333, and by the NSF grants number DMS-1109640 and DMS-1109645.

* Adam Larios alarios@unl.edu

† Mark R. Petersen mpetersen@lanl.gov

¥ Edriss S. Titi titi@math.tamu.edu and edriss.titi@weizmann.ac.il

$\S$ Beth Wingate B.Wingate@exeter.ac.uk

[1] A. Larios and E. S. Titi, Discrete Contin. Dyn. Syst. Ser. B 14, 603 (2010).

[2] A. Larios and E. Titi, (preprint) (2015), arXiv: 1507.08203.

[3] J. T. Beale, T. Kato, and A. J. Majda, Comm. Math. Phys. 94, 61 (1984).

[4] L. C. Berselli, T. Iliescu, and W. J. Layton, Mathematics of large eddy simulation of turbulent flows, Scientific Computation (Springer-Verlag, Berlin, 2006) pp. xviii+348.

[5] Y. Cao, E. Lunasin, and E. S. Titi, Commun. Math. Sci. 4, 823 (2006).

[6] A. P. Oskolkov, Zap. Naučn. Sem. Leningrad. Otdel. Mat. Inst. Steklov. (LOMI) 38, 98 (1973), boundary value problems of mathematical physics and related questions in the theory of functions, 7.

[7] A. P. Oskolkov, Zap. Nauchn. Sem. Leningrad. Otdel. Mat. Inst. Steklov. (LOMI) 115, 191 (1982), boundary value problems of mathematical physics and related questions in the theory of functions, 14 .

[8] M. Böhm, Math. Nachr. 155, 151 (1992).

[9] D. Catania, Ann. Univ Ferrara 56, 1 (2010), 10.1007/s11565-009-0069-1.

[10] D. Catania and P. Secchi, Quad. Sem. Mat. Univ. Brescia (2009).

[11] A. Larios, E. Lunasin, and E. S. Titi, (preprint) arXiv:1010.5024.

[12] M. A. Ebrahimi, M. Holst, and E. Lunasin, IMA J. App. Math. , 1 (2012), doi:10.1093/imamat/hxr069.

[13] B. Levant, F. Ramos, and E. S. Titi, Commun. Math. Sci. 8, 277 (2010).

[14] B. Khouider and E. S. Titi, Comm. Pure Appl. Math. 61, 1331 (2008).

[15] E. Olson and E. S. Titi, Nonlinear Anal. 66, 2427 (2007).

[16] F. Ramos and E. S. Titi, Discrete Contin. Dyn. Syst. 28, 375 (2010).

[17] V. K. Kalantarov, B. Levant, and E. S. Titi, J. Nonlinear Sci. 19, 133 (2009).

[18] V. K. Kalantarov and E. S. Titi, Chinese Ann. Math. B 30, 697 (2009).

[19] P. Kuberry, A. Larios, L. G. Rebholz, and N. E. Wilson, Comput. Math. Appl. 64, 2647 (2012).

[20] G. Di Molfetta, G. Krstlulovic, and M. Brachet, Phys. Rev. E 92, 013020 (2015).

[21] W. J. Layton and L. G. Rebholz, Int. J. Comput. Fluid Dyn. 27, 184 (2013).

[22] P. Constantin and C. Fefferman, Indiana Univ. Math. J. 42, 775 (1993).
[23] P. Constantin, C. Fefferman, and A. J. Majda, Comm. Partial Differential Equations 21, 559 (1996).

[24] J. D. Gibbon and E. S. Titi, J. Nonlinear Sci. 23, 993 (2013).

[25] A. B. Ferrari, Comm. Math. Phys. 155, 277 (1993).

[26] G. Ponce, Comm. Math. Phys. 98, 349 (1985).

[27] J. Deng, T. Y. Hou, and X. Yu, Comm. Partial Differential Equations 30, 225 (2005).

[28] T. Y. Hou, Acta Numer. 18, 277 (2009).

[29] T. Y. Hou and R. Li, in Mathematics and computation, a contemporary view, Abel Symp., Vol. 3 (Springer, Berlin, 2008) pp. 39-66.

[30] T. Y. Hou and R. Li, Phys. D 237, 1937 (2008).

[31] R. M. Kerr, Phys. Fluids A 5, 1725 (1993).

[32] J. D. Gibbon, Phys. D 237, 1894 (2008).

[33] A. J. Chorin, Math. Comp. 22, 745 (1968).

[34] R. Temam, Arch. Rational Mech. Anal. 32, 135 (1969).

[35] M. E. Brachet, D. Meiron, S. Orszag, B. Nickel, R. Morf, and U. Frisch, J. Statist. Phys. 34, 1049 (1984).

[36] M. E. Brachet, D. Meiron, S. Orszag, B. Nickel, R. Morf, and U. Frisch, J. Fluid Mech. 130, 411 (1983).

[37] T. B. Benjamin, J. L. Bona, and J. J. Mahony, Philos. Trans. Roy. Soc. London Ser. A 272, 47 (1972). 